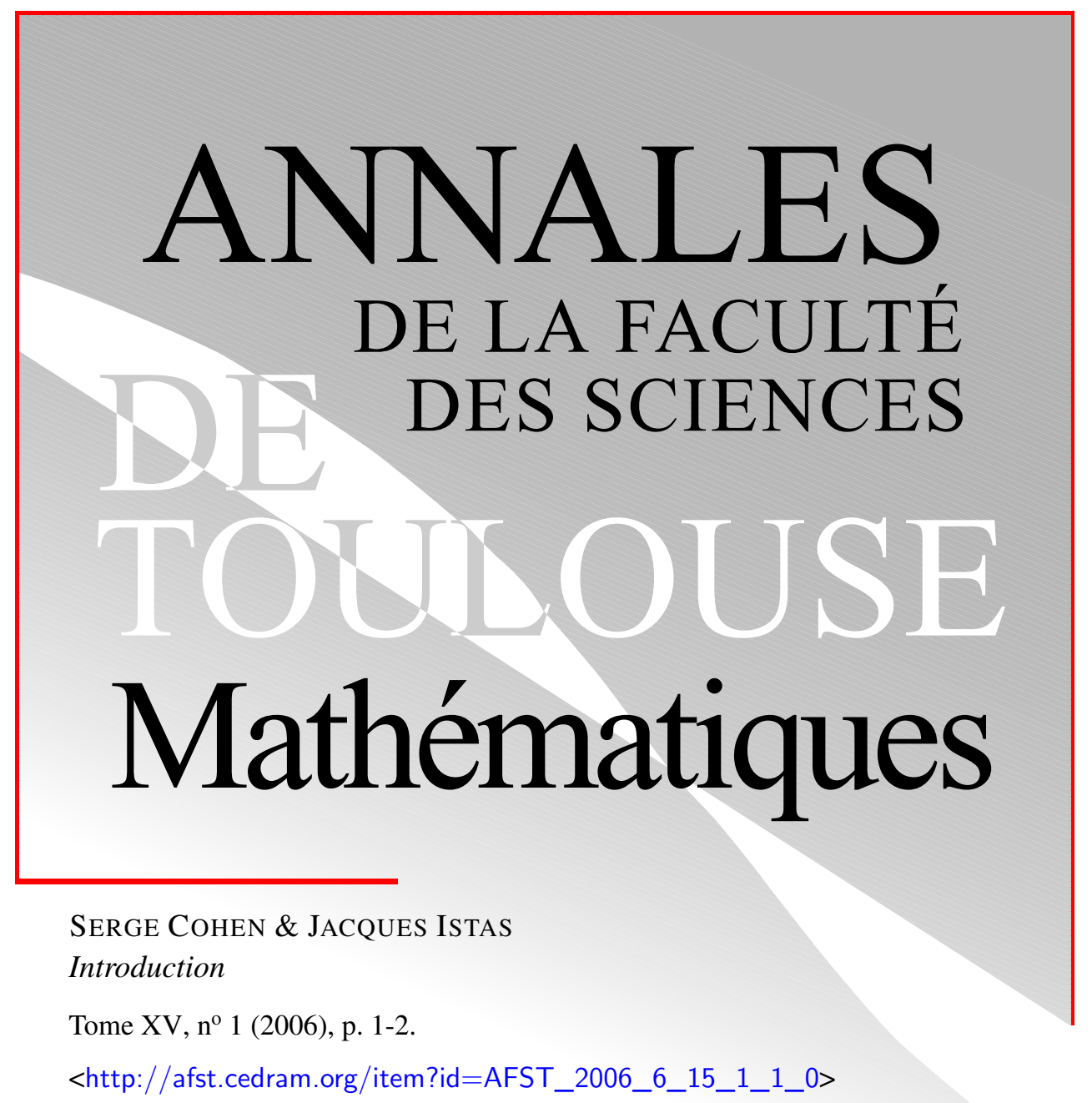

(C) Annales de la faculté des sciences de Toulouse Mathématiques, 2006, tous droits réservés.

L'accès aux articles de la revue "Annales de la faculté des sciences de Toulouse, Mathématiques » (http://afst.cedram.org/), implique l'accord avec les conditions générales d'utilisation (http://afst.cedram. org/legal/). Toute reproduction en tout ou partie cet article sous quelque forme que ce soit pour tout usage autre que l'utilisation à fin strictement personnelle du copiste est constitutive d'une infraction pénale. Toute copie ou impression de ce fichier doit contenir la présente mention de copyright.

\title{
cedram
}

Article mis en ligne dans le cadre du

Centre de diffusion des revues académiques de mathématiques

http://www.cedram.org/ 


\section{Second conference on self-similarity and applications}

Self-similarity arises from various problems in signal processing, stochastic calculus, turbulence, multifractal phenomena, among others. The aim of the Second Conference on Self-similarity and Applications, held in Toulouse from 20 to 24 of June 2005, was to offer a large overview on self-similarity and its applications. This special issue of the Annales de la Faculté des Sciences de Toulouse consists of research and expository papers of the invited talks. Let us briefly introduce these papers.

How can be formalized the idea that a function satisfies some scaling property in the neighbourhood of a given point? This question is seminal when working in a self-similar setting, and Stéphane Jaffard introduces and studies a general definition of pointwise regularity associated with a quasiBanach space which is shift and scaling invariant.

Since the Cantor set, deterministic or random trees have been used for modeling fractal sets. Jean-François Le Gall discusses the class of real selfsimilar random trees, which generalize the Continuous Random Trees, and explains their connections with superprocesses.

Stochastic calculus with respect to Brownian Motion is now a standard topic. Fractional Brownian Motion is the archetypal self-similar process and stochastic calculus with respect to it is an ongoing expansion theme. David Nualart provides us with a survey on different approaches introduced recently to construct stochastic integrals with respect to Fractional Brownian Motion: pathwise techniques, Malliavin calculus, approximation by Riemann sums.

Multiscale analysis of data is a usual tool in physics. Tools like wavelets and fractals are currently used and Sylvie Roques and Frédérique Rémi detail the multiscale analysis of solar cycles.

Long range dependence is a fascinant subject since B. Mandelbrot brought the attention of the scientific community to the water levels in the Nile river. Gennady Samorodnitsky gives the main ideas and results on long memory, scaling and self-similarity.

Many problems of statistical inference appear when working in a stochastic self-similar setting. Some of them are linked to the occupation measure of 
the path of the stochastic process. Mario Wschebor presents both classical and recent results on approximation theorems of the occupation measure.

Some questions, like small ball properties, Hausdorff dimensions and measures, require sharp estimates of sample path properties. Yimin Xiao shows how these properties should be linked to local nondeterministic selfsimilar stochastic processes.

At least, we hope that this issue provides a real survey on topical questions related to self-similarity.

Serge Cohen and Jacques Istas 